\title{
Coupling microwave photons to topological spin textures in $\mathrm{Cu}_{2} \mathrm{OSeO}_{3}$
}

\author{
S. Khan $\odot,{ }^{1,}{ }^{*}$ O. Lee $\odot,{ }^{1}$ T. Dion, ${ }^{1}$ C. W. Zollitsch $\odot,{ }^{1}$ S. Seki, ${ }^{2}$ Y. Tokura, ${ }^{2,3,4}$ J. D. Breeze $\odot,{ }^{5}$ and H. Kurebayashi ${ }^{1, \dagger}$ \\ ${ }^{1}$ London Centre for Nanotechnology, University College London, London WC1H OAH, United Kingdom \\ ${ }^{2}$ Department of Applied Physics, University of Tokyo, Tokyo 113-8656, Japan \\ ${ }^{3}$ RIKEN Center for Emergent Matter Science (CEMS), Wako 351-0198, Japan \\ ${ }^{4}$ Tokyo College, University of Tokyo, Tokyo 113-8656, Japan \\ ${ }^{5}$ Department of Materials, Imperial College London, Exhibition Road, London SW7 2AZ, United Kingdom
}

(Received 7 May 2021; revised 13 August 2021; accepted 23 August 2021; published 3 September 2021; corrected 8 September 2021)

\begin{abstract}
Topologically protected nanoscale spin textures, known as magnetic skyrmions, possess particlelike properties and feature emergent magnetism effects. In bulk cubic helimagnets, distinct skyrmion resonant modes are already identified using a technique such as ferromagnetic resonance in spintronics. However, direct light-matter coupling between microwave photons and skyrmion resonance modes still needs to be demonstrated. Utilizing two distinct cavity systems, we observe a direct interaction between the cavity resonant mode and two resonant skyrmion modes, the counterclockwise gyration and breathing modes, in bulk $\mathrm{Cu}_{2} \mathrm{OSeO}_{3}$. For both resonant modes, we find the largest coupling strength at $57 \mathrm{~K}$ indicated by an enhancement of the cavity linewidth at the degeneracy point. We study the effective coupling strength as a function of temperature within the expected skyrmion phase. We attribute the maximum in effective coupling strength to the presence of a large number of skyrmions, and correspondingly to a completely stable skyrmion lattice. Our experimental findings indicate that the coupling between photons and resonant modes of magnetic skyrmions depends on the relative density of these topological particles instead of the pure spin number in the system.
\end{abstract}

DOI: 10.1103/PhysRevB.104.L100402

\section{INTRODUCTION}

In condensed matter physics, the study of light-matter interactions holds an important stature as it provides physical insight into properties of coupled systems while facilitating the design of new-concept devices [1-3]. The light-matter coupling in this context between microwave photons residing in a cavity and particle ensembles has been studied in a wide range of material systems [4]. These systems include twodimensional electron gases [5,6], paramagnetic spins $[7,8]$, and exchange interaction dominated ordered spin systems [9-12]. Quantum information processing as quantum memories $[13,14]$ and quantum transducers [15] are particularly interesting applications which could be made possible due to the hybrid spin-ensemble-photon systems. The formation of a quasiparticle, known as the cavity-magnon polariton (CMP), results from the strong coherent coupling phenomenon between cavity photons and magnons (i.e., a quanta of spin waves) creating a hybridized state between the two [16]. One main advantage for using ferromagnetic materials as a base for spin-photon hybrid systems is the large spin number $(N)$, as this yields a very sizable collective coupling strength $\left(g_{\mathrm{c}}\right)$ between the two systems due to the scaling condition given as $g_{\mathrm{c}}=g_{0} \sqrt{N}$, where $g_{0}$ is the single spin-single photon coupling strength $[17,18]$.

\footnotetext{
*safe.khan.11@ucl.ac.uk

†h.kurebayashi@ucl.ac.uk
}

In our earlier work [19], we demonstrated the existence of a stable polariton state by achieving strong coupling between cavity photons and magnons in the field-polarized phase of $\mathrm{Cu}_{2} \mathrm{OSeO}_{3}$. We also observed dispersive coupling between cavity photons and helimagnons in the noncollinear phases (helical and conical phases). $\mathrm{Cu}_{2} \mathrm{OSeO}_{3}$ is a material system well known to host another exotic topologically protected spin texture state known as magnetic skyrmions [20], which stabilize as a skyrmion lattice in a narrow temperature region below the Curie temperature $T_{\mathrm{c}}$. It has been shown that the skyrmion lattice can be excited by magnetic resonance techniques [21,22], where the resonance comprises a collective skyrmion core precession in the counterclockwise (CCW) and clockwise directions (gyration modes), and the breathing mode where skyrmion cores periodically shrink and enlarge. An open question was left unanswered in our previous work [19]: What happens when the cavity photons try to couple with the excitations of a skyrmion lattice? The difficulty in answering this question was mainly due to the high cavity resonance frequency $(\approx 9 \mathrm{GHz})$, where a direct interaction with the skyrmion resonance modes $(\approx 1-2 \mathrm{GHz})$ could not be observed.

In this Letter, we designed two separate cavity systems, each optimized to explore the interaction between the cavity and the skyrmion excitation modes. Both cavities are designed to have their primary resonant modes within the expected frequency window of either the CCW or breathing skyrmion modes. We observe the onset of an avoided crossing of the cavity mode, indicating the interaction of the cavity with the skyrmion system by shifting the cavity mode significantly. 
(a) Interaction Concept

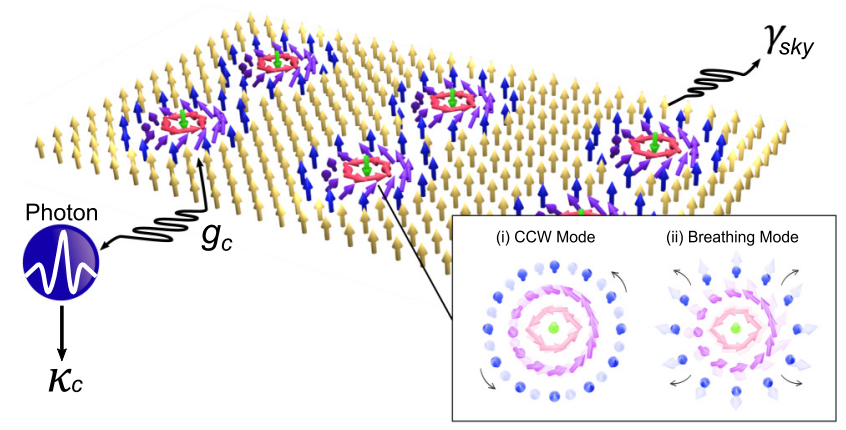

(b) CCW Mode

(c) Breathing Mode
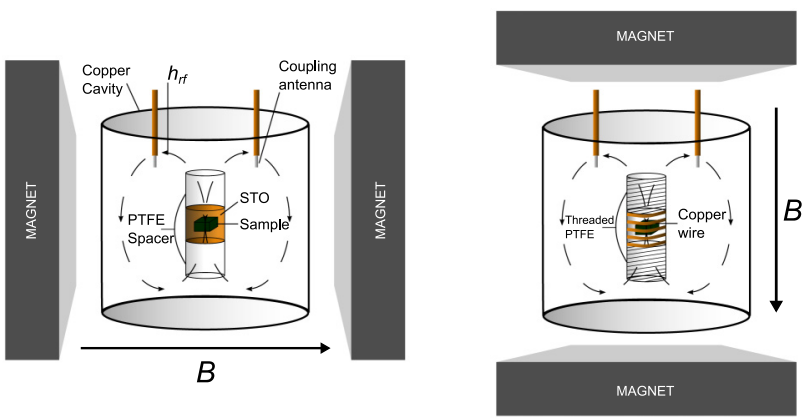

FIG. 1. (a) Illustration of the interaction between photons and excitation modes of the skyrmion lattice showing the resonant motions of (i) the CCW mode where the core of the skyrmion rotates, and (ii) the breathing mode which involves a periodic oscillation of the skyrmion core diameter. Schematic view of the experimental setup used with (b) the STO cavity where $\mathbf{h}_{\mathrm{rf}} \perp \mu_{0} \mathbf{H}$ for the CCW mode, and (c) the helical resonator where $\mathbf{h}_{\mathrm{rf}} \| \mu_{0} \mathbf{H}$ for the breathing mode. The cavity and resonator are held in a helium- 4 cryostat, and the cryostat sits in between the poles of the electromagnet. $\mathrm{A} \mathrm{Cu}_{2} \mathrm{OSeO}_{3}$ sample is placed inside the STO cavity and helical resonator with the support of polytetrafluoroethylene (PTFE) spacers and a threaded PTFE tube, respectively. The coupling to the cavity/resonator is achieved by connecting two microwave lines from the vector network analyzer (VNA) to the coupling loops in the copper cavity.

An enhancement in the cavity linewidth is observed at the degeneracy point, where cavity and skyrmion modes cross. By probing the cavity linewidth as a function of external magnetic field we are able to deduce the effective coupling strength as well as the effective loss rate of the skyrmion excitation. A detailed temperature dependence of the effective coupling strength is extracted for both the CCW and breathing skyrmion modes, indicating the presence of a large number of skyrmions in the vicinity of $57 \mathrm{~K}$. At this temperature the effective coupling strength is largest and we find the cavity-skyrmion hybrid system in the high cooperativity regime.

\section{EXPERIMENTAL PROCEDURE}

Figure 1(a) shows the schematic to highlight the interaction picture between photons (with a loss rate $\kappa_{\mathrm{c}}$ ) and skyrmion resonant modes (with an effective loss rate $\gamma_{\text {sky }}$ ), with $g_{\mathrm{c}}$ rep- resenting the effective coupling rate between the two excited systems. A dielectric strontium titanate (STO) and helical (helically wrapped copper wire) resonator are used to study the coupling of microwave photons with the counterclockwise $(\mathrm{CCW})$ and breathing skyrmion modes, respectively, in $\mathrm{Cu}_{2} \mathrm{OSeO}_{3}$. The remainder of Fig. 1 shows the experimental setup for the (b) STO and (c) helical resonator (see Supplemental Material for a full description [23]). The resonance frequency of the primary mode $\left(\mathrm{TE}_{01 \delta}\right)$ of the STO cavity, $\omega / 2 \pi \approx 1.1 \mathrm{GHz}$ at $57 \mathrm{~K}$, is in the expected range of the $\mathrm{CCW}$ excitation, as expected from broadband ferromagnetic resonance (FMR) measurements (see Supplemental Material) [21]. One can only excite the breathing mode when the microwave magnetic field in the cavity $\left(\mathbf{h}_{\mathrm{rf}}\right)$ is parallel to the external magnetic field $\left(\mu_{0} \mathbf{H}\right)$ [22], as shown in Fig. 1(c). This is achieved by exciting the primary mode of the helical resonator with an excitation frequency, $\omega / 2 \pi \approx 1.4 \mathrm{GHz}$. In the parallel excitation configuration, the excitation of all the other magnetic resonant modes in $\mathrm{Cu}_{2} \mathrm{OSeO}_{3}$ should be suppressed, and the resonator mode is expected only to couple to the breathing mode.

\section{RESULTS AND DISCUSSION}

\section{A. Onset of an avoided crossing effect in skyrmion phase}

Figure 2(a) shows the microwave transmission $\left|S_{21}\right|^{2}$ as a function of $\left(\mu_{0} \mathbf{H}\right)$ and probe frequency for the CCW skyrmion mode at $57 \mathrm{~K}$. The observed spectra exhibit a strong dependence of the cavity mode on $\mu_{0} \mathbf{H}$ and a clear shift in the resonance frequency is seen within the field region where a skyrmion lattice is expected. This shift represents an onset of an avoided crossing, due to the coupling of the cavity mode to the skyrmion excitations. A complete avoided crossing, where the cavity resonant mode splits into two peaks in frequency, indicates strong coupling [24]. The splitting corresponds to a hybridization of the two systems, where the separation relates to the coupling strength. The linewidth of the two peaks is defined by the average of the linewidth of the two subsystems. If the linewidth or loss rates of either one or both subsystems is larger than the coupling strength, the two peaks merge. This is the case for the cavity-skyrmion hybrid system we investigate here. In the following, we determine the effective coupling strength and effective skyrmion loss rate to classify the regime of coupling.

In Fig. 2(a), $\left|S_{21}\right|^{2}$ drops significantly in the field region between 150 and 400 Oe (i.e., in the expected field region

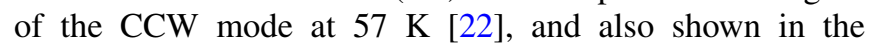
Supplemental Material through the coplanar waveguide FMR measurements). To study the evolution of the cavity resonance frequency and linewidth in greater detail, we fit a Lorentzian line shape for each magnetic field in the frequency domain [25]. Figure 2(b) shows the fitted spectrum at 1000 Oe and in the gap area $(300 \mathrm{Oe})$. The extracted resonance peak position is plotted (blue circles) in Fig. 2(a). A large increase in linewidth of the cavity mode is visible when $\mathrm{Cu}_{2} \mathrm{OSeO}_{3}$ is in the skyrmion phase. Note that we are observing a single broad line shape instead of two separated peaks, indicating that the hybrid system exhibits loss rates larger than the coupling strength [26]. 

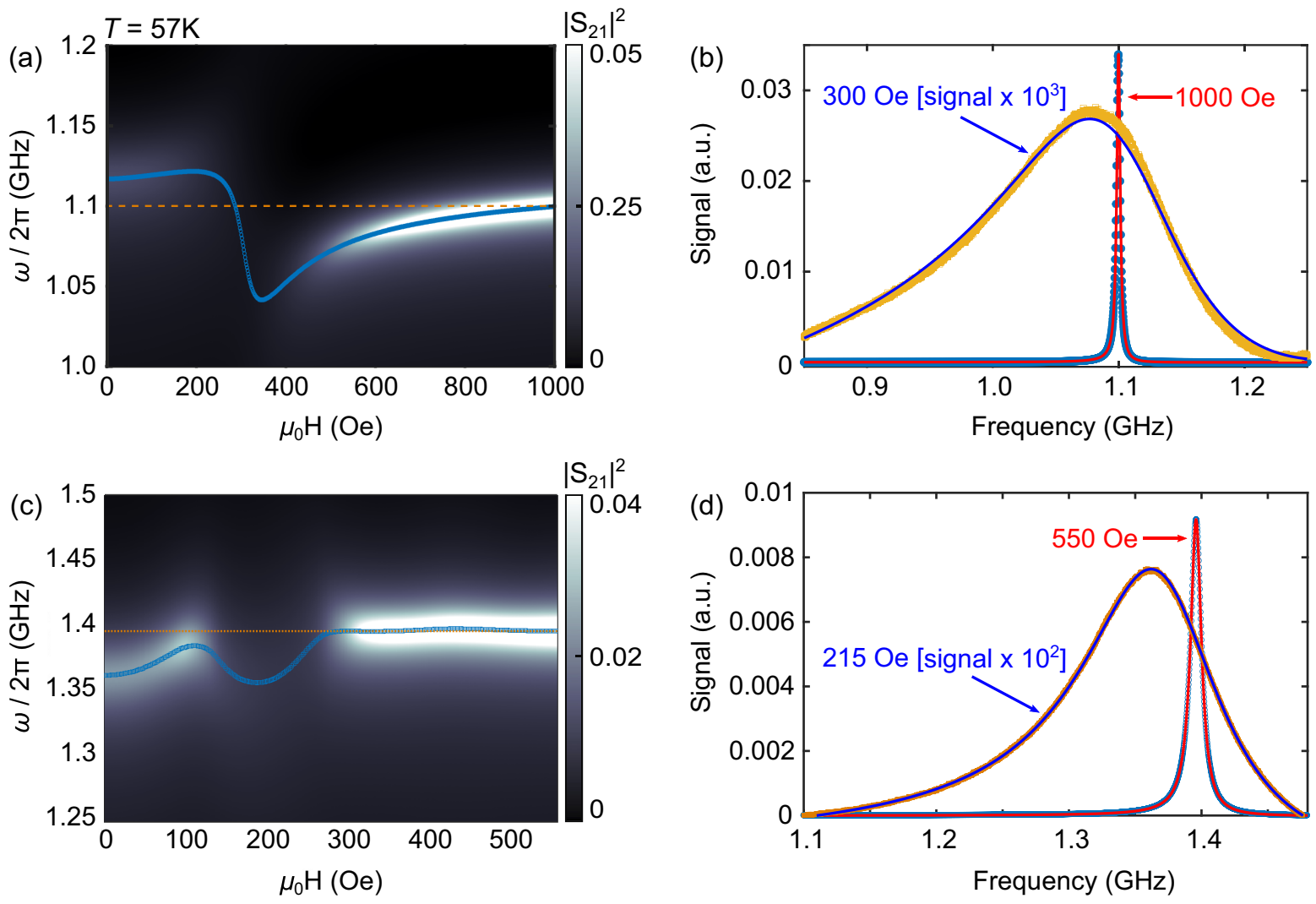

FIG. 2. The experimentally measured microwave transmission spectra $\left|S_{21}\right|^{2}$ at $57 \mathrm{~K}$ for (a) the CCW mode and (c) the breathing mode, where no background subtraction is performed for the data shown here. The dashed orange line represents the cavity mode, and the resonance peak position is plotted as circles (blue). (b) and (d) show line cuts in the frequency domain at high magnetic field away from skyrmion resonance and at a field region in the interaction point for the $\mathrm{CCW}$ and breathing mode, respectively. The data are fitted with a Lorentzian function.

Figure 2(c) shows the microwave transmission where the breathing skyrmion mode and the photon mode of the helical resonator interact at $57 \mathrm{~K}$. As in the case of the CCW skyrmion excitation mode, $\left|S_{21}\right|^{2}$ exhibits a decrease in intensity, in a magnetic field space covering the skyrmion phase. The microwave transmission as a function of probe frequency for magnetic fields on and far away from the skyrmion resonance is shown in Fig. 2(d). By following the same procedure of fitting a Lorentzian function in the frequency domain for each magnetic field, similarly to the $\mathrm{CCW}$ case, a clear resonance frequency shift and linewidth broadening are observed.

It is evident from Figs. 2(a) and 2(c) that the photon modes in the STO cavity and the helical resonator couple to the excitation modes of the skyrmion lattice, i.e., the CCW gyration and breathing mode, respectively. This is further supported by measurements at temperatures outside of the skyrmion phase $\left(T<T_{\text {sky }}<T_{\mathrm{c}}\right.$ and $\left.T>T_{\mathrm{c}}\right)$. Here, the cavity modes show no signature of coupling in resonance frequency or linewidth (respective color plots shown in the Supplemental Material [23]).

\section{B. Temperature dependence of coupling strength}

To identify the epitome of coupling between photons and skyrmion resonance, we analyzed the linewidth dependence of the cavity as a function of $\mu_{0} \mathbf{H}$ for both CCW and breathing modes. According to the two coupled harmonic oscillators model [12,27-30], the cavity linewidth (half width at half maximum) $\kappa_{(\mathrm{S} / \mathrm{H})}(\mathrm{S}$ : STO cavity and $\mathrm{H}$ : helical resonator) is given by the following Lorentzian profile, as

$$
\kappa_{(\mathrm{S} / \mathrm{H})}=\kappa_{\mathrm{c}}+\frac{g_{\mathrm{c}}^{2} \gamma_{\mathrm{sky}}}{\left(\Delta^{2}+\gamma_{\mathrm{sky}}^{2}\right)},
$$

where $\kappa_{\mathrm{c}}$ represents the loss rate of the cavity photons, $\gamma_{\mathrm{sky}}$ is the effective loss rate for skyrmion resonance, $g_{\mathrm{c}}$ is the effective coupling rate between the photons and skyrmion excitations, and $\Delta$ is the field-dependent detuning, given as $\Delta=\frac{g \mu_{0} \mu_{\mathrm{B}}}{\hbar}\left[\mathbf{H}-\mathbf{H}_{\text {res }}\right]$, with $g$ being the $g$-factor of the sample, taken as $g=2.1$ for $\mathrm{Cu}_{2} \mathrm{OSeO}_{3}$ [22].

Figures 3(a) and 3(b) show the evolution of the extracted linewidth as a function of $\mu_{0} \mathbf{H}$ at different temperatures for the CCW and breathing skyrmion modes, respectively. It is clearly noticeable that in both cases $\kappa_{(\mathrm{S} / \mathrm{H})}$ shows a peaklike behavior. At high magnetic fields, the photon mode in the STO cavity and the helical resonator is decoupled from the magnetic system and the measured linewidth mainly remains constant. A large deviation in the linewidth is observed as the magnetic field approaches the expected field region for the skyrmion lattice in the phase diagram at given temperatures. For temperatures outside of the skyrmion phase, i.e., 
(a)

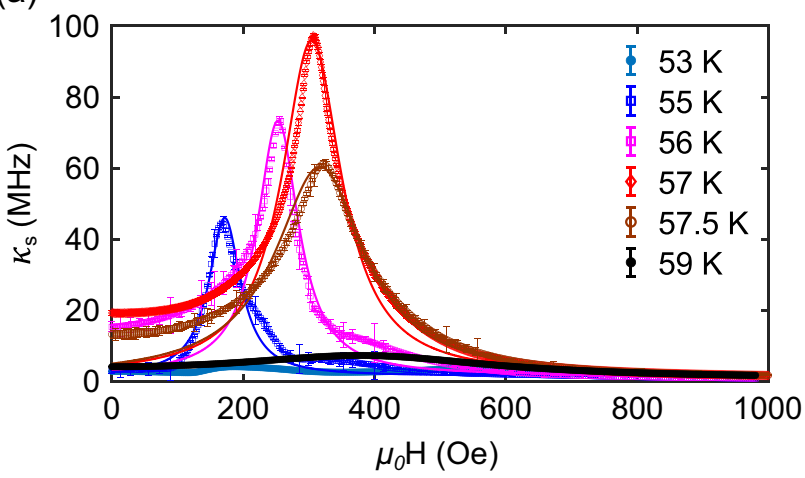

(b)

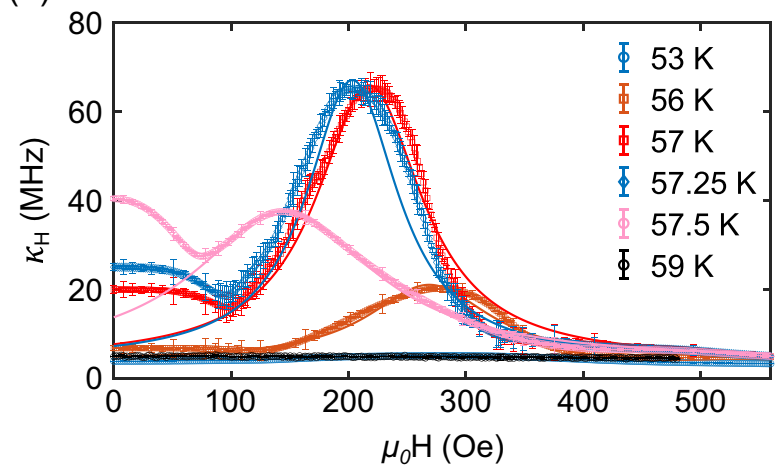

FIG. 3. Experimentally extracted linewidth (HWHM) of (a) the STO cavity interacting with the CCW mode and (b) helical resonator coupling to the breathing mode as a function of external magnetic field for different temperatures. The data in both are fitted with Eq. (1) in the main text.

$53 \mathrm{~K}\left(T<T_{\text {sky }}\right)$ and $59 \mathrm{~K}\left(T>T_{\mathrm{c}}\right)$, we observe no significant change in linewidth as can be seen in Figs. 3(a) and 3(b). This provides strong evidence that the photon mode couples to the skyrmion resonance modes.

In the skyrmion phase, for both the $\mathrm{CCW}$ and breathing modes, a fit to the data in Figs. 3(a) and 3(b) is performed using Eq. (1) with $\kappa_{\mathrm{c}}, \gamma_{\mathrm{sky}}$, and $g_{\mathrm{c}}$ as free parameters. The largest effective coupling strength is observed at $57 \mathrm{~K}$ for the CCW and breathing skyrmion modes. The extracted fitting parameters at $57 \mathrm{~K}$ are $g_{\mathrm{c}}^{\mathrm{ccw}} / 2 \pi=118 \mathrm{MHz}, \gamma_{\text {sky }} / 2 \pi=250 \mathrm{MHz}$, and $\kappa_{\mathrm{c}}^{\text {sto }} / 2 \pi=1.5 \mathrm{MHz}$ for the CCW mode, and $g_{\mathrm{c}}^{\mathrm{br}} / 2 \pi=$ $108 \mathrm{MHz}, \gamma_{\text {sky }} / 2 \pi=309 \mathrm{MHz}$, and $\kappa_{\mathrm{c}}^{\text {heli }} / 2 \pi=4 \mathrm{MHz}$ for the breathing mode.

The type of coupling observed between photons and skyrmion resonance modes falls in a regime where $\kappa_{\mathrm{c}}<g_{\mathrm{c}}<$ $\gamma_{\text {sky }}$ for both the CCW and breathing modes. The skyrmion resonance is heavily damped such that the rate of excitation loss happens at a faster rate so that the energy cannot be transferred back and forth between the photon and skyrmion systems. This is illustrated in the schematic shown as an inset in Fig. 4, where the driven photon system $(|10\rangle)$ coupled to the skyrmion resonant mode $(|01\rangle)$ opens up an additional path back to the ground state $(|00\rangle)$. This results in an enhancement of the linewidth $\left(\kappa_{(\mathrm{S} / \mathrm{H})}\right)$ of the microwave cavity due to its coupling to the skyrmion resonant modes.

Figure 4 shows the temperature dependence of $g_{\mathrm{c}}$ extracted from the linewidth analysis for both $\mathrm{CCW}$ and breathing skyrmion modes. As mentioned earlier, in $\mathrm{Cu}_{2} \mathrm{OSeO}_{3}$, the skyrmion phase is expected to exist in a narrow temperature range below $T_{\mathrm{c}}$. We see that for both the CCW and breathing modes, the highest effective coupling strength is observed for a $1 \mathrm{~K}$ range centered around $57 \mathrm{~K}$. The effective coupling strength gradually falls down when the system is further cooled, and no coupling is observed at $53 \mathrm{~K}$ where the linewidth of the STO cavity and the helical resonator becomes unaffected. The experimentally observed trend in the temperature dependence of the effective coupling strength is asymmetric. Above $57.5 \mathrm{~K}$, there is a sharp decrease in $g_{\mathrm{c}}$, reflective of a transition from an ordered magnetic state to the paramagnetic state.
It is well established that the coupling between photons and an ensemble of spins scales with the square root of the thermally polarized number of spins $(N)$ involved in the interaction, $g_{\mathrm{c}} \propto \sqrt{N}$ [31]. We anticipate that for interpreting the results shown in Fig. 4, one has to think of the coupling between photons and skyrmion resonance being dependent on the effective number of skyrmions taking part in the interaction. This suggests that around $57 \mathrm{~K}$, a stable hexagonal skyrmion lattice is present, resulting in a greater number of skyrmions. As we move away from this centered region of the skyrmion phase, the number of skyrmions decrease, resulting

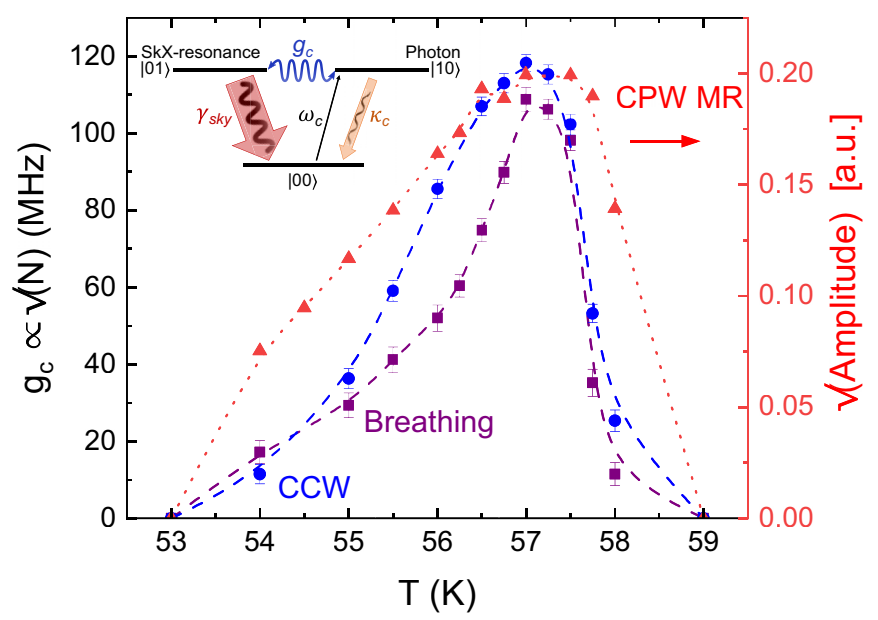

FIG. 4. The left $y$ axis shows the effective coupling strength $g_{\mathrm{c}}$ as a function of temperature for the $\mathrm{CCW}$ mode (blue circles) and breathing mode (purple squares). The right $y$ axis shows the temperature dependence of the square-root amplitude of resonance peaks extracted from the CPW magnetic resonance experiment. The inset shows a schematic illustrating an interaction between photons and skyrmion excitation. $|00\rangle$ corresponds to the ground state where no photons or skyrmion excitation exist in the cavity: When driven by microwave power, the cavity enters the excited state $|10\rangle$. Coupling of $|10\rangle$ with the excited skyrmion state $|01\rangle$ with coupling strength $g_{c}$ increases the cavity loss rate by having an additional damped channel through skyrmions. The dashed lines are guides for the eye. 
in a mixed state of magnetic domains and skyrmions. Hence, we see a decrease in the coupling strength between photons and skyrmion resonance. This is further supported by the temperature dependence of the intensity of small-angle neutron scattering (SANS) and the relaxation rate of muon-spin relaxation measurements in $\mathrm{Cu}_{2} \mathrm{OSeO}_{3}$ [32-34], where the intensity and relaxation rate, respectively, have a maximum around $57 \mathrm{~K}$ and a drop was observed at different temperatures in the skyrmion phase.

In order to further elaborate on the above interpretation, we carried out coplanar waveguide (CPW) magnetic resonance measurements in the skyrmion phase on the same sample used for the cavity experiments (see Supplemental Material [23]). The intensity ( $I \propto$ amplitude) of the magnetic resonance peak is proportional to the magnetization in the system [35]. In turn, probing the intensity, here mainly governed by the skyrmion population and the effective amplitude of the local magnetic moment under thermal fluctuation, in the skyrmion phase of $\mathrm{Cu}_{2} \mathrm{OSeO}_{3}$ should be an indicator of the effective number of skyrmions contributing to the magnetic resonance peak amplitude at any given temperature. Therefore, the temperature dependence of the square-root amplitude of the magnetic resonance peaks can reflect the trend seen in the effective coupling strength, i.e., $g_{\mathrm{c}} \propto \sqrt{N}$. Figure 4 (right $y$ axis) shows the square root of the amplitude of CPW magnetic resonance peaks as a function of $T$. The maximum value of the amplitude is observed in the vicinity of $57 \mathrm{~K}$, and is qualitatively similar to the trend observed in $g_{\mathrm{c}}$ for a narrow temperature region where a stable hexagonal skyrmion lattice in $\mathrm{Cu}_{2} \mathrm{OSeO}_{3}$ is expected.

In cavity QED, the cooperativity is a dimensionless figure of merit for the coupling strength given as $C=g_{\mathrm{c}}^{2} / \kappa_{\mathrm{c}} \gamma_{\text {sky }}$ [12], with $C>1$ being a condition to achieve coherent coupling. In this work, the largest effective coupling strength value results in $C=37$ for the coupling of STO cavity photons to the CCW skymion mode and $C=10$ for the coupling between the helical resonator photons and the breathing skyrmion mode. In both cases, an unstable polariton state is created between photons and skyrmion resonance, coined as a regime of high cooperativity. At resonance nearly all photons inside the cavity are coherently transferred into skyrmion excitations, but transferring the excitations back will require a skyrmion resonance system with a smaller loss rate (i.e., $\gamma_{\text {sky }}<g_{\text {c }}$ ). An earlier theoretical study on the coupling of photons to a breathing skyrmion mode in a thin magnetic disk does conclude that reaching the strong coupling condition in such a system is challenging due to the large loss rate of the skyrmion modes [36].

In conclusion, we have probed the type of coupling between photons in either a STO cavity or a helical resonator to the $\mathrm{CCW}$ and breathing skyrmion modes in $\mathrm{Cu}_{2} \mathrm{OSeO}_{3}$, respectively. At resonance, an onset of avoided crossing effect is observed, indicating an interaction between the photon mode and skyrmion modes. An enhancement in linewidth at the degeneracy point reveals that the system is in a regime of high cooperativity with $\kappa_{\mathrm{c}}<g_{\mathrm{c}}<\gamma_{\text {sky }}$ for both the CCW and breathing modes. The effective coupling strength exhibits a distinct dependence on temperature within the skyrmion phase, and comparing it with the amplitude of CPW magnetic resonance measurements confirms the presence of a skyrmion lattice with a maximum effective number of skyrmions within the vicinity of $57 \mathrm{~K}$. For photon-magnon interactions, the coupling strength scales with the square root of the number of thermally polarized spins/particles. This study reveals that the interaction between photons and resonant modes of topological spin structures strongly depends on the density of these topological particles instead of the pure spin number in the system. It seems challenging to theoretically predict the number of skyrmions in a bulk system such as $\mathrm{Cu}_{2} \mathrm{OSeO}_{3}$, with difficulty in an accurate determination of the temperature dependence of internal demagnetization fields, anisotropy fields, exchange and Dzyaloshinskii-Moriya interactions, and taking into account the quantum effects from spins. The support of a reliable theory can allow this coupling technique to be used in the future as an experimental determination of the number of skyrmions in a given system.

Note added. Recently, we became aware of a study of dispersive coupling, away from the resonance frequency of skyrmion excitations, between the skyrmion modes and cavity photon mode, with a high cooperativity observed at $56.5 \mathrm{~K}$ [37].

\section{ACKNOWLEDGMENTS}

The research was supported by JSPS Grants-In-Aid for Scientific Research (Grants No. 18H03685, No. 20H00349, and No. 21H04440), JST PRESTO (Grant No. JPMJPR18L5), Asahi Glass Foundation, and JST CREST (Grant No. JPMJCR1874). We would like to thank J. Langdon (UCL EEE workshop) for making the parts to place the cavity system inside of the cryostat. We are grateful to Professor M. Mochizuki for helpful discussions.
[1] Y. Li, W. Zhang, V. Tyberkevych, W.-K. Kwok, A. Hoffmann, and V. Novosad, J. Appl. Phys. 128, 130902 (2020).

[2] A. Clerk, K. Lehnert, P. Bertet, J. Petta, and Y. Nakamura, Nat. Phys. 16, 257 (2020).

[3] D. D. Awschalom, C. Du, R. He, F. Heremans, A. Hoffmann, J. Hou, H. Kurebayashi, Y. Li, L. Liu, V. Novosad et al., IEEE Trans. Quantum Eng. (2021).

[4] B. Bhoi and S.-K. Kim, Solid State Phys. 70, 1 (2019).

[5] V. M. Muravev, I. V. Andreev, I. V. Kukushkin, S. Schmult, and W. Dietsche, Phys. Rev. B 83, 075309 (2011).
[6] G. Scalari, C. Maissen, D. Turčinková, D. Hagenmüller, S. De Liberato, C. Ciuti, C. Reichl, D. Schuh, W. Wegscheider, M. Beck et al., Science 335, 1323 (2012).

[7] I. Chiorescu, N. Groll, S. Bertaina, T. Mori, and S. Miyashita, Phys. Rev. B 82, 024413 (2010).

[8] J. D. Breeze, E. Salvadori, J. Sathian, N. M. Alford, and C. W. Kay, npj Quantum Inf. 3, 40 (2017).

[9] Y. Tabuchi, S. Ishino, T. Ishikawa, R. Yamazaki, K. Usami, and Y. Nakamura, Phys. Rev. Lett. 113, 083603 (2014). 
[10] X. Zhang, C.-L. Zou, L. Jiang, and H. X. Tang, Phys. Rev. Lett. 113, 156401 (2014).

[11] M. Goryachev, W. G. Farr, D. L. Creedon, Y. Fan, M. Kostylev, and M. E. Tobar, Phys. Rev. Appl. 2, 054002 (2014).

[12] H. Huebl, C. W. Zollitsch, J. Lotze, F. Hocke, M. Greifenstein, A. Marx, R. Gross, and S. T. B. Goennenwein, Phys. Rev. Lett. 111, 127003 (2013).

[13] R. Schoelkopf and S. Girvin, Nature (London) 451, 664 (2008).

[14] G. Kurizki, P. Bertet, Y. Kubo, K. Mølmer, D. Petrosyan, P. Rabl, and J. Schmiedmayer, Proc. Natl. Acad. Sci. U.S.A. 112, 3866 (2015).

[15] S. Blum, C. O'Brien, N. Lauk, P. Bushev, M. Fleischhauer, and G. Morigi, Phys. Rev. A 91, 033834 (2015).

[16] M. Harder and C.-M. Hu, Solid State Phys. 69, 47 (2018).

[17] G. S. Agarwal, Phys. Rev. Lett. 53, 1732 (1984).

[18] A. Imamoğlu, Phys. Rev. Lett. 102, 083602 (2009).

[19] L. V. Abdurakhimov, S. Khan, N. A. Panjwani, J. D. Breeze, M. Mochizuki, S. Seki, Y. Tokura, J. J. L. Morton, and H. Kurebayashi, Phys. Rev. B 99, 140401(R) (2019).

[20] Y. Okamura, F. Kagawa, M. Mochizuki, M. Kubota, S. Seki, S. Ishiwata, M. Kawasaki, Y. Onose, and Y. Tokura, Nat. Commun. 4, 2391 (2013).

[21] Y. Onose, Y. Okamura, S. Seki, S. Ishiwata, and Y. Tokura, Phys. Rev. Lett. 109, 037603 (2012).

[22] T. Schwarze, J. Waizner, M. Garst, A. Bauer, I. Stasinopoulos, H. Berger, C. Pfleiderer, and D. Grundler, Nat. Mater. 14, 478 (2015).

[23] See Supplemental Material at http://link.aps.org/supplemental/ 10.1103/PhysRevB.104.L100402 for the detailed experimental method, VNA calibration, cavity measurements outside of the skyrmion phase, and the CPW magnetic resonance experiment.

[24] D. Lachance-Quirion, Y. Tabuchi, A. Gloppe, K. Usami, and Y. Nakamura, Appl. Phys. Express 12, 070101 (2019).
[25] S. S. Kalarickal, P. Krivosik, M. Wu, C. E. Patton, M. L. Schneider, P. Kabos, T. J. Silva, and J. P. Nibarger, J. Appl. Phys. 99, 093909 (2006).

[26] L. Bai, K. Blanchette, M. Harder, Y. Chen, X. Fan, J. Xiao, and C.-M. Hu, IEEE Trans. Magn. 52, 1 (2016).

[27] S. Haroche and J.-M. Raimond, Exploring the Quantum: Atoms, Cavities, and Photons (Oxford University Press, Oxford, UK, 2006).

[28] P. F. Herskind, A. Dantan, J. P. Marler, M. Albert, and M. Drewsen, Nat. Phys. 5, 494 (2009).

[29] E. Abe, H. Wu, A. Ardavan, and J. J. Morton, Appl. Phys. Lett. 98, 251108 (2011).

[30] G. Dold, C. W. Zollitsch, J. O'Sullivan, S. Welinski, A. Ferrier, P. Goldner, S. E. de Graaf, T. Lindström, and J. J. L. Morton, Phys. Rev. Appl. 11, 054082 (2019).

[31] C. W. Zollitsch, K. Mueller, D. P. Franke, S. T. Goennenwein, M. S. Brandt, R. Gross, and H. Huebl, Appl. Phys. Lett. 107, 142105 (2015).

[32] T. Adams, A. Chacon, M. Wagner, A. Bauer, G. Brandl, B. Pedersen, H. Berger, P. Lemmens, and C. Pfleiderer, Phys. Rev. Lett. 108, 237204 (2012).

[33] S. Seki, J.-H. Kim, D. S. Inosov, R. Georgii, B. Keimer, S. Ishiwata, and Y. Tokura, Phys. Rev. B 85, 220406(R) (2012).

[34] T. J. Hicken, M. N. Wilson, K. J. A. Franke, B. M. Huddart, Z. Hawkhead, M. Gomilšek, S. J. Clark, F. L. Pratt, A. Štefančič, A. E. Hall, M. Ciomaga Hatnean, G. Balakrishnan, and T. Lancaster, Phys. Rev. B 103, 024428 (2021).

[35] M. Farle, Rep. Prog. Phys. 61, 755 (1998).

[36] M. J. Martínez-Pérez and D. Zueco, New J. Phys. 21, 115002 (2019).

[37] L. Liensberger, F. X. Haslbeck, A. Bauer, H. Berger, R. Gross, H. Huebl, C. Pfleiderer, and M. Weiler, arXiv:2102.11713.

Correction: The previously published versions of Figures 2(a) and 2(c) contained plotting errors and their revisions have been posted. 